\title{
The Role of MeCP2 in Brain Development and Neurodevelopmental Disorders
}

\author{
Michael L. Gonzales • Janine M. LaSalle
}

Published online: 24 March 2010

(C) The Author(s) 2010. This article is published with open access at Springerlink.com

\begin{abstract}
Methyl $\mathrm{CpG}$ binding protein-2 (MeCP2) is an essential epigenetic regulator in human brain development. Rett syndrome, the primary disorder caused by mutations in the $\mathrm{X}$-linked MECP2 gene, is characterized by a period of cognitive decline and development of hand stereotypies and seizures following an apparently normal early infancy. In addition, MECP2 mutations and duplications are observed in a spectrum of neurodevelopmental disorders, including severe neonatal encephalopathy, X-linked mental retardation, and autism, implicating $\mathrm{MeCP} 2$ as an essential regulator of postnatal brain development. In this review, we compare the mutation types and inheritance patterns of the human disorders associated with $M E C P 2$. In addition, we summarize the current understanding of $\mathrm{MeCP} 2$ as a central epigenetic regulator of activity-dependent synaptic maturation. As $\mathrm{MeCP} 2$ occupies a central role in the pathogenesis of multiple neurodevelopmental disorders, continued investigation into MeCP2 function and regulatory pathways may show promise for developing broad-spectrum therapies.
\end{abstract}

Keywords Rett syndrome · Angelman's syndrome ·

Autism $\cdot$ Epigenetic $\cdot$ Neurodevelopmental

\section{Introduction}

The MECP2 gene encodes methyl $\mathrm{CpG}$ binding protein-2 (MeCP2), a transcriptional regulator required for proper

M. L. Gonzales · J. M. LaSalle $(\bowtie)$

School of Medicine, Medical Microbiology and Immunology, University of California, Davis,

One Shields Avenue,

Davis, CA 95616, USA

e-mail: jmlasalle@ucdavis.edu

M. L. Gonzales

e-mail: migonzales@ucdavis.edu neuronal development. Mutations in $M E C P 2$ are the major cause of the neurodevelopmental disorder Rett syndrome (RTT), an X-linked disorder that primarily affects females. $M E C P 2$ mutations and dysfunctions have also been associated with a broad array of other neurodevelopmental disorders in males and females, including X-linked mental retardation (XLMR), severe neonatal encephalopathy, Angelman's syndrome (AS), and autism, demonstrating that disruption of MeCP2 can have wide-ranging effects on neurodevelopment and lead to a spectrum of disorders [1]. However, the extent of $M E C P 2$ mutations within these individual disorders is variable. Although $M E C P 2$ mutations can account for up to $96 \%$ of classic RTT [2], they are rare in other disorders (eg, autism), indicating that MECP2 is just one of many genes that could contribute to the broader spectrum of neurodevelopmental phenotypes. Additionally, a clear correlation does not always exist between specific $M E C P 2$ mutations and individual disorders or phenotypic severity, suggesting that genetic, epigenetic, and/or environmental factors interact with the $M E C P 2$ mutation to determine the final phenotypic outcome in terms of type and severity of the disorder.

$M E C P 2$ mutations can be grouped into three general categories: severe loss-of-function mutations, mild loss-offunction mutations, and a broad group of duplications and other noncoding mutations that affect $\mathrm{MeCP} 2$ expression levels. Each mutation type is associated with a subset of disorders (Fig. 1). The involvement of MeCP2 in multiple neurodevelopmental disorders demonstrates that it occupies a central role in the postnatal development of the human brain. Understanding the role that $\mathrm{MeCP} 2$ plays in brain development and maturation is important for understanding the pathogenesis of these disorders and identifying potential therapeutic targets. To achieve this will require a thorough understanding of the molecular mechanisms through which $\mathrm{MeCP} 2$ acts, as well as how $\mathrm{MeCP} 2$ interacts with 


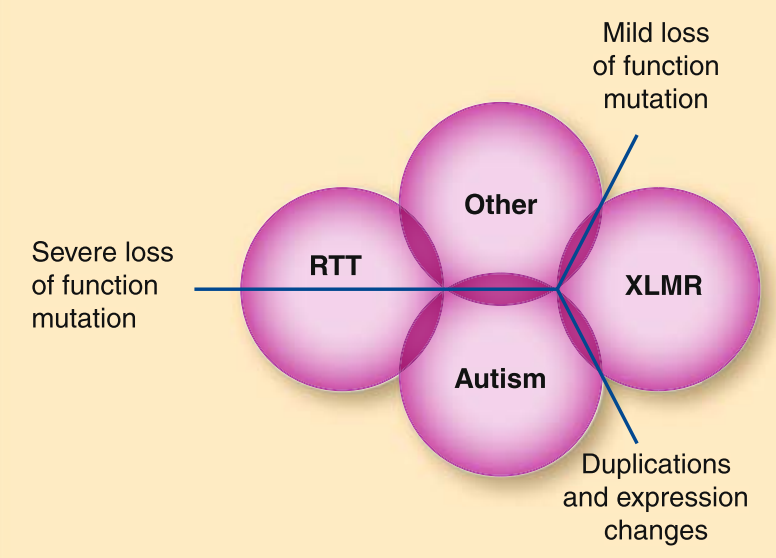

Fig. 1 Association of neurodevelopmental disorders with specific classes of Methyl CpG binding protein-2 (MeCP2) mutation. The schematic diagram depicts the major MeCP2-based neurodevelopmental disorders and their association with each class of MeCP2 mutation. RTT - Rett syndrome; XLMR - X-linked mental retardation

signaling and epigenetic pathways in the developing brain.

\section{MECP2 Inheritance and X Inactivation}

MECP2 is located on the $\mathrm{X}$ chromosome. As such, it is subjected to an $\mathrm{X}$-linked inheritance pattern. Females inherit two X chromosomes, one maternal and one paternal, whereas males inherit their single $\mathrm{X}$ chromosome maternally. Therefore, whereas females can inherit $M E C P 2$ mutations from either parent, male inheritance of $M E C P 2$ mutations is exclusively maternal. This has dramatic effects on the transmittance of $M E C P 2$ mutations as well as the stratification of $M E C P 2$-associated disorders by gender (Table 1). The MECP2 gene is also subject to $\mathrm{X}$ chromosome inactivation (XCI) in females. $\mathrm{XCI}$ is the process by which one of the two $\mathrm{X}$ chromosomes carried by females is inactivated to achieve gene expression patterns similar to those found in males, who carry only one copy of the X chromosome. XCI occurs on a cell-by-cell basis and is a predominantly random process. However, extreme degrees of skewing can occur with preferential inactivation of the maternal or paternal $\mathrm{X}$ chromosome. Therefore, the phenotypic results of $\mathrm{MeCP} 2$ mutations in females are likely not only based on the type of mutation but also on the degree of inactivation between the mutant and wild-type alleles. With a single $\mathrm{X}$ chromosome, males are more severely affected by $M E C P 2$ mutations than females, who along with the mutant gene on one $\mathrm{X}$ chromosome carry a normal copy of $M E C P 2$ on the other.

$M E C P 2$ mutations are mostly sporadic, occurring preferentially as $\mathrm{C}>\mathrm{T}$ transitions of $\mathrm{CpG}$ dinucleotides and preferentially on the paternal $\mathrm{X}$ chromosome [3, 4]. It has been postulated that the low number of males with $M E C P 2$ mutations is the result of a lethal in utero effect of having no functional $M E C P 2$ from their single $\mathrm{X}$ chromosome. However, the predominantly paternal origin of MeCP2 mutations likely prevents them from being transmitted to male offspring in the first place.

\section{MeCP2 and Neurodevelopmental Disorders}

\section{Rett Syndrome}

MeCP2 dysfunction is associated with several neurodevelopmental disorders. The primary neurodevelopmental disorder associated with $M E C P 2$ mutations is RTT, which affects about 1 in 15,000 females [5]. Classic RTT is characterized by apparently normal early development for the first 6 to 18 months of life followed by a period of developmental regression and loss of acquired skills that results in intellectual disability (ID), loss of speech and purposeful hand movements, ataxia, seizures, and respiratory abnormalities. There are several recognized variants of RTT in

Table 1 Neurodevelopmental disorders associated with MeCP2 mutations

\begin{tabular}{|c|c|c|c|c|}
\hline Studies & Disorder & Mutation & Gender & $\begin{array}{l}\text { Parent of } \\
\text { origin }\end{array}$ \\
\hline Amir et al. [5] & Rett syndrome & Severe loss of function & Female & Paternal \\
\hline Schanen et al. [8], Villard et al. [9] & Severe neonatal encephalopathy & Severe loss of function & Male & Maternal \\
\hline Carney et al. [21] & Autism & Severe loss of Function & Female & Paternal $(?)$ \\
\hline Couvert et al. [18] & $\mathrm{X}$-linked mental retardation & Mild loss of function & Male & Maternal \\
\hline Lugtenberg et al. [19•] & X-linked mental retardation & Duplication & Male & Maternal \\
\hline $\begin{array}{l}\text { Loat et al. [22], Nagarajan et al. [23], } \\
\text { Shibayama et al. [24], Ramocki et al. [25••] }\end{array}$ & Autism & $\begin{array}{l}\text { Duplication/noncoding } \\
\text { variants }\end{array}$ & Male & Maternal \\
\hline $\begin{array}{l}\text { Adegbola et al. [27], Cohen et al. [28], } \\
\text { Watson et al. [29] }\end{array}$ & Other & Severe/mild & Male/female & Either (?) \\
\hline
\end{tabular}

$M e C P 2$ methyl $\mathrm{CpG}$ binding protein-2 
addition to the classic form, including the congenital, earlyonset seizure, and preserved speech variants, which have similar yet distinct clinical phenotypes from classic RTT. About $96 \%$ of classic RTT cases are associated with MECP2 mutations [2], whereas other forms are largely associated with other genetic mutations, such as CDKL5 in the earlyonset seizure variant [6] and FOXG1 mutations in the congenital variant [7•]. Thus, whereas MECP2 mutations are clearly responsible for most classic RTT, they are not the only genetic lesion that can yield RTT phenotypes.

Significant phenotypic variability exists in classic RTT, even among individuals with the same MECP 2 mutation. Skewed XCI has been used to explain the mild phenotype associated with rare maternal carriers of $M E C P 2$ mutations $[8,9]$. In addition, three different Mecp2-deficient murine models showed variably skewed XCI favorably inactivating the mutant allele and correlating with phenotypic severity $[10,11]$. However, most girls with classic RTT show random X chromosome inactivation patterns [12]. Furthermore, recent studies have questioned the exclusive ability of XCI status to explain discordance between individuals with identical $M E C P 2$ mutations but different severity, suggesting the influence of genetic modifiers $[13 \bullet, 14 \bullet]$.

\section{Severe Neonatal Encephalopathy}

The classic RTT phenotype in males is exceedingly rare and associated with a 47,XXY karyotype or somatic mosaicism for an $M E C P 2$ mutation $[15,16]$. Both of these situations recapitulate the female scenario of XCI and allow for expression of some wild-type $M E C P 2$. In karyotypically normal boys, RTT-associated MECP2 mutations present as severe neonatal encephalopathy, a disorder characterized by a static encephalopathy, severe developmental delays, hypotonia, seizures, and respiratory abnormalities that often leads to death at an early age [8, 9]. Most of these individuals inherited the $M E C P 2$ mutation from mildly or unaffected mothers with favorable XCI skewing [8, 9]. Because XCI of the mutant $M E C P 2$ allele is random in most females, favorably skewed maternal carriers of RTTassociated mutations are rare. Therefore, the prevalence of severe neonatal encephalopathy caused by $M E C P 2$ mutations is also thought to be rare. However, most boys with this disorder have been identified because they have female siblings with RTT. It is possible that more cases exist outside of known RTT families that have yet to be identified.

\section{X-linked Mental Retardation}

XLMR is a source of inherited ID affecting 1 in 600 to 1 in 1000 males, as well as a smaller-but still significantnumber of females [17]. XLMR is a genetically diverse disorder arising from mutation or duplication of genes across the $\mathrm{X}$ chromosome, including $M E C P 2$. In early studies, $M E C P 2$ point mutations were identified in up to $2 \%$ of individuals with XLMR [18]. In males, these mutations result in severe ID, speech impairment, and motor abnormalities - including progressive spasticitywhereas females display mild ID or appear unaffected. To date, XLMR-associated MECP2 mutations have not been identified in RTT or severe neonatal encephalopathy; also, RTT mutations have not been found in cases of XLMR. Duplications in Xq28, a region of the $\mathrm{X}$ chromosome that includes $M E C P 2$, also have been implicated in $1 \%$ to $2 \%$ of XLMR cases [19॰]. Analysis of these duplications in multiple XLMR families has identified a region containing $M E C P 2$ and its neighbor IRAK1 as the minimal genomic region associated with XLMR and Xq28 duplications [19•]. $M E C P 2$ duplication syndrome is characterized by severe ID, lack of speech, progressive spasticity, and susceptibility to respiratory infections in males and mildly affected or unaffected females. The molecular situation arising from $M E C P 2$ duplication is very different from that caused by $M E C P 2$ mutations in XLMR. Whereas mutations are thought to cause neurodevelopmental disorders through reduced levels of functional $\mathrm{MeCP} 2, \mathrm{MeCP} 2$ duplications actually result in elevated MeCP2 levels [20]. That both under- and overexpression of functional $\mathrm{MeCP} 2$ can lead to neurodevelopmental disorders serves to highlight the requirement for correct regulation of $\mathrm{MeCP} 2$ expression for proper neuronal development.

Female carriers of XLMR MECP2 mutations generally show balanced XCI, signifying that XLMR mutations are less severe compared with those that result in RTT. Alternatively, MECP2 duplication carrier females display extreme XCI skewing ( $85 \%-100 \%)$ favoring inactivation of the duplicated allele [20]. This suggests that the effects of $M E C P 2$ duplication are more severe than those of the XLMR mutations and that the mild phenotype in these females is due to preferential inactivation of the duplicated allele. Both the relatively mild nature of XLMR mutations in females and the preferential XCI skewing of duplications allow for maternal transmission of defective $M E C P 2$ alleles to male and female offspring $[19 \bullet, 20]$, a situation that is not possible with the more severe RTT mutations. Because of this, MECP 2 mutations associated with XLMR are much more common in males than those associated with RTT.

Autism

Autism and RTT have significant phenotypic overlap, and both are classified as pervasive developmental disorders. Although autism and RTT have a strong genetic component, the genetic basis of autism is unclear and likely involves multiple genes. Mutations in MECP2 identical to some classic RTT mutations have been identified in a small 
number of autistic females who do not meet the diagnostic criteria for RTT [21]. In addition to discrete MECP2 mutations, linkage analysis has identified the over- and undertransmittance of specific $M E C P 2$ variants in families with autistic children, suggesting a link between MECP2 and increased susceptibility to autism [22]. Reduced expression of $\mathrm{MeCP} 2$ protein frequently occurs in the frontal cortex of autistic individuals and is correlated with increased MECP2 promoter methylation [23]. Decreased $\mathrm{MeCP} 2$ expression may also be caused by variations in 3 regulatory elements of $M E C P 2$, which are found more commonly in autistic individuals than in controls [24]. Finally, a recent study found a high comorbidity of autism in males with $M E C P 2$ duplications inherited from maternal carriers with neuropsychiatric symptoms $[25 \bullet \bullet]$. Therefore, as with XLMR, over- and underexpression of MeCP2 can lead to an autistic phenotype in some individuals.

Autism is relatively rare in girls, making the association of $M E C P 2$ mutations in girls with an autistic phenotype particularly interesting. Because of the phenotypic overlap between RTT and autism, as well as the phenotypic variability between individuals in both disorders, it is not clear if an MECP2 mutation with an autism diagnosis is a different disorder than RTT, or if the two are simply different representations on a spectrum associated with $M E C P 2$ mutations. It was reported that an initial diagnosis of autism in girls with MECP2 mutations was not maintained as they grew older, and it was suggested that the autistic phenotype may actually represent an RTT variant [26]. Regardless of whether another autistic phenotype associated with $M E C P 2$ mutations is truly autism, it is likely that the phenotypic differences from RTT or XLMR arise from differences in genetic background and environmental exposures in each affected individual.

\section{Other Disorders}

MECP2 mutations also have been reported in other neurological cases. A father and daughter were identified with a mild neurobehavioral phenotype including cognitive and motor difficulties. Both carry a proline-to-alanine point mutation at amino acid 152 in $\mathrm{MeCP} 2$, and the daughter displays balanced XCI, suggesting that this mutation has a mild effect on MeCP2 function [27]. It is interesting to note that a different mutation at the same site (proline to arginine) is commonly found in classic RTT. The arginine mutation has a much more dramatic effect on the biochemical properties of $\mathrm{MeCP} 2$ than does the alanine mutation, which is likely related to the differences in phenotypic severity between the two mutations [27]. Another report described a boy with early-onset schizophrenia and developmental receptive language disorder with a mutation in MECP2 [28]. This same mutation is found in XLMR but presents with a vastly different phenotype in this case. Cases of AS with $M E C P 2$ mutations also have been reported [29]. AS is a neurodevelopmental disorder with some phenotypic similarities to RTT but with an earlier onset characterized by hypotonicity at birth. AS is primarily caused by deletions, mutations, or imprinting errors of the UBE3A gene on chromosome 15, but $15 \%$ of AS cases have no identified genetic abnormality at this site. Several different MECP2 mutations, most of which are also found in RTT, have been found in boys and girls diagnosed with AS. Some individuals transitioned to a more RTT-like phenotype with age, whereas others maintained an AS-like phenotype. The appearance of MECP2 mutations usually associated with other disorders in these cases is another demonstration of variable expressivity. In these cases, rare genetic variants and/or environmental insults may interact with the $M E C P 2$ mutations to produce the uncommon phenotypes described previously. Therefore, not only is there extensive variability of phenotypes associated with single mutations within specific disorders, but the capacity also exists for specific mutations to give rise to distinct disorders in different individuals.

\section{MeCP2 Function}

$\mathrm{MeCP} 2$ is a member of a family of proteins that specifically bind to methylated DNA sequences in the genome. DNA methylation is a covalent modification to $\mathrm{CpG}$ dinucleotides and an epigenetic mark used by cells to alter expression of genes without permanently changing the DNA sequence. In this way, DNA methylation represents a mitotically heritable yet reversible modification to the genome. By binding to methylated DNA, MeCP2 can read these epigenetic marks in the genome and translate them into functional effects of modified gene expression.

Historically, DNA methylation has been thought to be a repressive mark, primarily when found at $\mathrm{CpG}$-rich gene promoters. Therefore, $\mathrm{MeCP} 2$ was predicted to be a global repressor of transcription of genes by binding to methylated gene promoters and recruiting transcriptional repressors to silence gene expression [30]. However, gene promoters are vastly de-enriched for DNA methylation, and global analyses of MeCP2-dependent expression have not yielded many clear target genes with densely methylated promoters that require $\mathrm{MeCP} 2$ for silencing, suggesting that the function of $\mathrm{MeCP} 2$ is broader than the proximal repression of methylated gene promoters. Recently, new models of $\mathrm{MeCP} 2$ function have been proposed in which MeCP2 acts as a transcriptional modulator capable of regulating increases and decreases in expression of transcriptionally active genes by long-range regulation of chromatin structure from distal methylation sites outside of gene promoters $[31,32 \cdot]$. An additional study suggested an activating role 
for $\mathrm{MeCP} 2$ by its interaction with the transcription factor CREB at the promoters of active genes [33•].

Many gene targets of $\mathrm{MeCP} 2$ undergo rapid changes in expression following activity-dependent neuronal activation. In the case of the MeCP2 target gene $B D N F$, this is achieved through activity-dependent phosphorylation of $\mathrm{MeCP} 2$ [34]. By acting as an activity-dependent regulator of gene expression, $\mathrm{MeCP} 2$ is positioned to integrate signals from neuronal activation and epigenetic marks to control the expression of its target genes. It is likely that the heterogeneous nature of activity-dependent activation and epigenetic status of individual neurons or neuronal networks in the maturing brain have led to the inability to fully delineate the entire spectrum of MeCP2 target genes.

$\mathrm{MeCP} 2$ is ubiquitously expressed in human tissues, but particularly high protein levels are found in the brain [35]. Within the brain, neurons contain the highest levels of $\mathrm{MeCP} 2$, but expression in these cells is heterogeneous and based primarily on the maturational state of the individual neurons. The level of MeCP2 in neurons is initially low and increases over the course of postnatal neuronal development, reaching a maximum in a subpopulation of mature postmitotic neurons [36]. This pattern of expression suggests that $\mathrm{MeCP} 2$ is involved in the maturation of existing neurons rather than the development of new neurons from precursor cells. Imaging studies have shown that girls with $M E C P 2$ mutations and RTT have an overall reduction in brain volume compared with controls [37], but there have been no reports of major neuropathological abnormalities in girls with RTT. Instead, MECP2 mutations seem to result in subtle changes in neuronal morphology. Neuroanatomic studies demonstrate that specific populations of neurons from girls with RTT are smaller and have reduced dendritic complexity than those found in control samples, adding support to the idea that $\mathrm{MeCP} 2$ is involved in neuronal maturation as opposed to formation. Although $\mathrm{MeCP} 2$ expression is highest in neurons, glial cells have detectable levels of MeCP2 expression as well. Recent reports have suggested that $\mathrm{MeCP} 2$ expression in astrocytes may be critical for RTT pathogenesis by disrupting support for neuronal dendritic maturation [38•, 39•].

Murine models have been developed by targeted disruption of Mecp2 that recapitulates many of the neurodevelopmental phenotypes seen in humans [40-42]. Deficiency of MeCP2 specifically in postmitotic neurons is responsible for the neurodevelopmental deficits in these mice $[40,43]$. Combined with the lack of major anatomic defects in neurons lacking functional $\mathrm{MeCP} 2$, this suggests that a lack of MeCP2 does not result in irreversible damage to neurons. Instead, $\mathrm{MeCP} 2$ mutations may cause a premature arrest in neuronal development with relative sparing of neuronal commitment. Accordingly, reintroduction of functional $\mathrm{MeCP} 2$, even in symptomatic mice, can rescue the developmental phenotypes associated with MeCP2 deficiency [44•, 45••]. However, it is important to note that overexpression of $\mathrm{MeCP} 2$ by even twofold can cause related severe neurological defects [43, 46], indicating that levels of MeCP2 are tightly regulated and that too little as well as too much functional $\mathrm{MeCP} 2$ can cause neurodevelopmental disorders. The sensitivity of neurons to aberrant levels of MeCP2 means that attempts to rescue $\mathrm{MeCP} 2$ dysfunction in humans through reintroduction of a functional $M E C P 2$ transgene will be challenging and require a deeper understanding of $\mathrm{MeCP} 2$ 's regulation and mechanism(s) of action.

\section{Molecular Mechanisms of Disease}

Although much progress has been made in understanding the functions of MeCP2 in recent years, it is still unclear exactly how MECP2 mutations contribute to the pathogenesis of RTT and related syndromes. The major anatomic differences in brains of both girls with RTT and Mecp2-deficient mice are reduced dendritic arborization and dendritic spine formation, both of which are indicative of immature neurons and would be predicted to represent a deficit in synaptic formation and/or transmission. In support of this hypothesis, it has been shown that brain samples from Mecp2-deficient mice display reduced spontaneous synaptic transmission as well as reduced synaptic plasticity - molecular processes that underlie synaptic formation, learning, and memory [47]. Reduction of spontaneous transmission and synaptic plasticity in these mice is a result of reduced synaptic connectivity and a weakening of the remaining neuronal connections caused by loss of Mecp2 [48•]. Synaptic deficits also have been reported in transgenic mice with twofold Mecp2 overexpression [46], again demonstrating the strict requirements for regulation of $\mathrm{MeCP} 2$ levels in neuronal maturation. Synapse formation and maturation requires activitydependent gene expression in neurons. Activation of an immature neuron causes the induction of a large number of genes that help strengthen and mature the developing synapse. MeCP2 controls the expression of several of these genes, including $B D N F, I D 1, E G R 2$, and JUNB. Activitydependent phosphorylation of $\mathrm{MeCP} 2$ is required for the activity-dependent expression of BDNF [34] and proper dendritic branching and dendritic spine formation in neurons. Therefore, a loss of MeCP2-regulated, activity-dependent gene expression may lead to the widespread deficiencies in synaptic maturation observed in the brains of Mecp2-null mice and girls with RTT. This in turn is thought to be the underlying cause of RTT and other MeCP2-associated neurodevelopmental disorders. The late-infancy onset of RTT symptoms corresponds to the occurrence of elevated $\mathrm{MeCP} 2$ levels in maturing neurons and fits with the predicted 
role for MeCP2 in activity-dependent postnatal maturation of neuronal synapses.

A large number of $M E C P 2$ mutations have been reported, including point mutations that change single amino acids in $\mathrm{MeCP} 2$, truncations that cause premature termination that results in shortened $\mathrm{MeCP} 2$ protein, and small deletions that are missing stretches of amino acids from the interior of MeCP2. Mutations can be found throughout the protein but tend to cluster in or include regions that are important for $\mathrm{MeCP} 2$ functions. Through mutation of critical amino acids or deletion of important functional domains, MECP2 mutations result in a loss of $\mathrm{MeCP} 2$ function. Mutant proteins show a reduced affinity for methylated DNA and a reduced ability to regulate gene expression [49]. Because of this, MeCP2 mutants cannot properly regulate activity-dependent gene expression in response to neuronal stimulation. Alternatively, MECP2 duplication or other mutations in noncoding regions of the gene can lead to changes in MeCP2 expression levels and do not change the intrinsic functions of the protein. Instead, abnormal levels of MeCP2 will result in misregulation of $\mathrm{MeCP} 2$ target genes. Both situations lead to improper modulation of MeCP2-regulated pathways and result in deficient neuronal maturation.

MECP2 mutations are involved in many different disorders with substantial phenotypic overlap (Fig. 1). In some instances, there is a clear segregation of specific $M E C P 2$ mutations and particular disorders. MECP2 mutations found in RTT are rarely seen in XLMR and vice versa. This difference is due to the effects of different types of mutations on MeCP2 function. Mutations found in XLMR have a relatively mild effect on the biochemical functions of MeCP2 when compared with those found in RTT [50]. This is likely to result in different patterns of neuronal maturation and dysfunction, which will lead to different phenotypic outcomes. However, a clear segregation between mutation type and symptoms is not always apparent. Identical MECP2 mutations can be found in individuals with many different diagnoses, ranging from classic RTT to autism to AS. Even within RTT, a large phenotypic variability exists between individuals with the same MECP 2 mutation. Some of this variability can be attributed to differences in mutation type and XCI, but these alone cannot explain all the variability seen in RTT. Much of this variability across disorders and within RTT is likely due to genetic modifiers - that is, variants of genes that are a part of MeCP2-regulated pathways. On their own, variations in these genes may not lead to visible phenotypes, but they can affect the phenotypic outcome of MECP2 mutations. It has been reported that a common polymorphism in the $B D N F$ gene found in healthy and affected individuals correlates with increased severity and susceptibility to seizures in RTT [51•]. Additional genetic modifiers likely have yet to be identified, many of which may have no apparent effect on their own but when combined with mutations in $M E C P 2$ can lead to different neurodevelopmental phenotypes. Therefore, it is likely a complex interaction between mutation type, XCI skewing (in the case of females), and other genetic modifiers that leads to the final phenotype in $M E C P 2$ mutations.

\section{Conclusions}

As is apparent by the spectrum and severity of associated disorders, MeCP2 seems to be a critical regulator of neuronal activity-dependent synaptic maturation. To achieve this, $\mathrm{MeCP} 2$ integrates epigenetic marks and signals from neuronal activation to regulate the gene expression patterns required for neuronal maturation through long-range and local regulation of chromatin structure. As such, MeCP2 occupies a central role in the postnatal development of the human brain. However, MeCP2 does not work in isolation. It is influenced by and acts upon countless other genetic, epigenetic, and environmental factors, all of which work in concert to insure proper development of the human brain. Although each of these $M E C P 2$-associated disorders is distinct, they share significant phenotypic overlap, including core deficits in cognition and motor function. A more complete understanding of the molecular mechanisms surrounding $\mathrm{MeCP} 2$, as well as the pathways in which it resides will not only enhance our understanding of neuronal maturation but will help us to understand the pathogenesis of multiple neurodevelopmental diseases. The relative sparing of neuronal commitment in $M E C P 2$-related disorders provides hope that potential therapies to restore $M E C P 2$ function or reactivate $\mathrm{MeCP} 2$-dependent pathways may provide significant benefit to individuals suffering from these disorders.

Disclosure No potential conflicts of interest relevant to this article were reported.

Open Access This article is distributed under the terms of the Creative Commons Attribution Noncommercial License which permits any noncommercial use, distribution, and reproduction in any medium, provided the original author(s) and source are credited.

\section{References}

Papers of particular interest, published recently, have been highlighted as:

- Of importance

•• Of major importance

1. Hammer S, Dorrani N, Dragich J, et al.: The phenotypic consequences of MECP2 mutations extend beyond Rett syndrome. Ment Retard Dev Disabil Res Rev 2002, 8:94-98. 
2. Zoghbi HY: MeCP2 dysfunction in humans and mice. J Child Neurol 2005, 20:736-740.

3. Girard M, Couvert P, Carrie A, et al.: Parental origin of de novo MECP2 mutations in Rett syndrome. Eur J Hum Genet 2001, 9:231-236.

4. Trappe R, Laccone F, Cobilanschi J, et al.: MECP2 mutations in sporadic cases of Rett syndrome are almost exclusively of paternal origin. Am J Hum Genet 2001, 68:1093-1101.

5. Amir RE, Van den Veyver IB, Wan M, et al.: Rett syndrome is caused by mutations in X-linked MECP2, encoding methyl- CpGbinding protein 2. Nat Genet 1999, 23:185-188.

6. Lin C, Franco B, Rosner MR: CDKL5/Stk9 kinase inactivation is associated with neuronal developmental disorders. Hum Mol Genet 2005, 14:3775-3786.

7. - Ariani F, Hayek G, Rondinella D, et al.: FOXG1 is responsible for the congenital variant of Rett syndrome. Am J Hum Genet 2008, 83:89-93. Investigating patients with a congenital variant of RTT that show evidence of hypotonia and developmental delay from birth, the investigators found novel mutations in the autosomal gene FOXG1, encoding a transcription factor with expression restricted to fetal and adult brain and testes. MeCP2 and FOXG1 might share common molecular mechanisms because of their similar expression patterns and nuclear localization.

8. Schanen NC, Kurczynski TW, Brunelle D, et al.: Neonatal encephalopathy in two boys in families with recurrent Rett syndrome. J Child Neurol 1998, 13:229-231.

9. Villard L, Kpebe A, Cardoso C, et al.: Two affected boys in a Rett syndrome family: clinical and molecular findings. Neurology 2000, 55:1188-1193.

10. Braunschweig D, Simcox T, Samaco RC, LaSalle JM: Xchromosome inactivation ratios affect wild-type MeCP2 expression within mosaic Rett syndrome and Mecp2-/+ mouse brain. Hum Mol Genet 2004, 13:1275-1286.

11. Young JI, Zoghbi HY: X-chromosome inactivation patterns are unbalanced and affect the phenotypic outcome in a mouse model of Rett syndrome. Am J Hum Genet 2004, 74:511-520.

12. Shahbazian MD, Sun Y, Zoghbi HY: Balanced X chromosome inactivation patterns in the Rett syndrome brain. Am J Med Genet 2002, 111:164-168.

13. - Takahashi S, Ohinata J, Makita Y, et al.: Skewed X chromosome inactivation failed to explain the normal phenotype of a carrier female with MECP2 mutation resulting in Rett syndrome. Clin Genet 2008, 73:257-261. This was an unusual case report describing the maternal transmission of an RTT-causing MECP2 mutation and skewed XCI; however, the mother's preferentially inactivated allele was the wild-type allele and could not explain the apparently normal phenotype of the mother.

14. - Bao X, Jiang S, Song F, et al.: X chromosome inactivation in Rett syndrome and its correlations with MECP2 mutations and phenotype. J Child Neurol 2008, 23:22-25. This was a thorough investigation of XCI in RTT phenotype in 52 RTT cases, 50 RTT mothers, and 48 control females showing that XCI skewing and genotype are insufficient to explain the phenotypic manifestations of RTT.

15. Leonard H, Silberstein J, Falk R, et al.: Occurrence of Rett syndrome in boys. J Child Neurol 2001, 16:333-338.

16. Topcu M, Akyerli C, Sayi A, et al.: Somatic mosaicism for a MECP2 mutation associated with classic Rett syndrome in a boy. Eur J Hum Genet 2002, 10:77-81.

17. Gecz J, Shoubridge C, Corbett M: The genetic landscape of intellectual disability arising from chromosome X. Trends Genet 2009, 25:308-316.

18. Couvert P, Bienvenu T, Aquaviva C, et al.: MECP2 is highly mutated in X-linked mental retardation. Hum Mol Genet 2001, 10:941-946.

19. - Lugtenberg D, Kleefstra T, Oudakker AR, et al.: Structural variation in Xq28: MECP2 duplications in $1 \%$ of patients with unexplained XLMR and in $2 \%$ of male patients with severe encephalopathy. Eur J Hum Genet 2009, 17:444-453. (Published erratum appears in Eur J Hum Genet 2009, 17:697.) MECP2 duplications were found in about 1\% of male patients with unexplained XLMR and as many as $2 \%$ of patients with severe progressive neurologic symptoms. In contrast, no MECP2 duplications were observed in 329 female patients.

20. Van Esch H, Bauters M, Ignatius J, et al.: Duplication of the MECP2 region is a frequent cause of severe mental retardation and progressive neurological symptoms in males. Am J Hum Genet 2005, 77:442-453.

21. Carney RM, Wolpert CM, Ravan SA, et al.: Identification of $\mathrm{MeCP} 2$ mutations in a series of females with autistic disorder. Pediatr Neurol 2003, 28:205-211.

22. Loat CS, Curran S, Lewis CM, et al.: Methyl-CpG-binding protein 2 polymorphisms and vulnerability to autism. Genes Brain Behav 2008, 7:754-760.

23. Nagarajan RP, Hogart AR, Gwye Y, et al.: Reduced MeCP2 expression is frequent in autism frontal cortex and correlates with aberrant MECP2 promoter methylation. Epigenetics 2006, 1:e1-e11.

24. Shibayama A, Cook EH Jr, Feng J, et al.: MECP2 structural and 3'-UTR variants in schizophrenia, autism and other psychiatric diseases: a possible association with autism. Am J Med Genet B Neuropsychiatr Genet 2004, 128B:50-53.

25. •• Ramocki M, Sarika UP, Tavyev YJ, et al.: Autism and other neuropsychiatric symptoms are prevalent in individuals with MECP2duplication syndrome. Ann Neurology 2009, 66:771782. This was a thorough clinical and neuropsychiatric assessment of affected boys with MECP2 mutations and their carrier mothers showing that all boys with MECP2 duplication boys had autism, whereas female carriers showed a variety of psychiatric symptoms despite exhibiting skewed XCI.

26. Zappella M, Meloni I, Longo I, et al.: Study of MECP2 gene in Rett syndrome variants and autistic girls. Am J Med Genet B Neuropsychiatr Genet 2003, 119B:102-107.

27. Adegbola AA, Gonzales ML, Chess A, et al.: A novel hypomorphic MECP2 point mutation is associated with a neuropsychiatric phenotype. Hum Genet 2009, 124:615-623.

28. Cohen D, Lazar G, Couvert P, et al.: MECP2 mutation in a boy with language disorder and schizophrenia. Am J Psychiatry 2002, 159:148-149.

29. Watson P, Black G, Ramsden S, et al.: Angelman syndrome phenotype associated with mutations in MECP2, a gene encoding a methyl CpG binding protein. J Med Genet 2001, 38:224-228.

30. Jones PL, Veenstra GJ, Wade PA, et al.: Methylated DNA and $\mathrm{MeCP} 2$ recruit histone deacetylase to repress transcription. Nat Genet 1998, 19:187-191.

31. Horike S, Cai S, Miyano M, et al.: Loss of silent-chromatin looping and impaired imprinting of DLX5 in Rett syndrome. Nat Genet 2005, 37:31-40.

32. • Yasui DH, Peddada S, Bieda MC, et al.: Integrated epigenomic analyses of neuronal MeCP2 reveal a role for long-range interaction with active genes. Proc Natl Acad Sci U S A 2007, 104:19416-19421. This ChIP-chip and epigenomic analysis of $\mathrm{MeCP} 2$ binding sites in tiled genomic regions and promoters genome wide challenged the expectation that MeCP2 would be primarily bound to inactive gene promoters.

33. - Chahrour M, Jung SY, Shaw C, et al.: MeCP2, a key contributor to neurological disease, activates and represses transcription. Science 2008, 320:1224-1229. This study showed that MeCP2 associates with the transcriptional activator CREB1 and for most identified target genes activated rather than repressed expression.

34. Zhou Z, Hong EJ, Cohen S, et al.: Brain-specific phosphorylation of $\mathrm{MeCP} 2$ regulates activity-dependent BDNF transcription, dendritic growth, and spine maturation. Neuron 2006, 52:255-269.

35. Shahbazian MD, Antalffy B, Armstrong DL, Zoghbi HY: Insight into Rett syndrome: MeCP2 levels display tissue- and cell-specific 
differences and correlate with neuronal maturation. Hum Mol Genet 2002, 11:115-124.

36. Balmer D, Goldstine J, Rao YM, LaSalle JM: Elevated methylCpG-binding protein 2 expression is acquired during postnatal human brain development and is correlated with alternative polyadenylation. J Mol Med 2003, 81:61-68.

37. Carter JC, Lanham DC, Pham D, et al.: Selective cerebral volume reduction in Rett syndrome: a multiple-approach MR imaging study. AJNR Am J Neuroradiol 2008, 29:436-441.

38. - Ballas N, Lioy DT, Grunseich C, Mandel G: Non-cell autonomous influence of MeCP2-deficient glia on neuronal dendritic morphology. Nat Neurosci 2009, 12:311-317. This study showed that MeCP2 is expressed in astrocytes, and glial $\mathrm{MeCP} 2$ deficiency contributed to neuronal dendritic morphology defects in a murine model of RTT.

39. - Maezawa I, Swanberg S, Harvey D, et al.: Rett syndrome astrocytes are abnormal and spread MeCP2 deficiency through gap junctions. J Neurosci 2009, 29:5051-5061. This study also showed glial expression and the importance of astrocytes in RTT pathogenesis by spreading MeCP2 deficiency through gap junctions.

40. Chen RZ, Akbarian S, Tudor M, Jaenisch R: Deficiency of methyl-CpG binding protein-2 in CNS neurons results in a Rettlike phenotype in mice. Nat Genet 2001, 27:327-331.

41. Guy J, Hendrich B, Holmes M, et al.: A mouse Mecp2-null mutation causes neurological symptoms that mimic Rett syndrome. Nat Genet 2001, 27:322-326.

42. Shahbazian M, Young J, Yuva-Paylor L, et al.: Mice with truncated $\mathrm{MeCP} 2$ recapitulate many Rett syndrome features and display hyperacetylation of histone H3. Neuron 2002, 35:243254.

43. Luikenhuis S, Giacometti E, Beard CF, Jaenisch R: Expression of $\mathrm{MeCP} 2$ in postmitotic neurons rescues Rett syndrome in mice. Proc Natl Acad Sci U S A 2004, 101:6033-6038.

44. - Giacometti E, Luikenhuis S, Beard C, Jaenisch R: Partial rescue of MeCP2 deficiency by postnatal activation of MeCP2. Proc Natl Acad Sci U S A 2007, 104:1931-1936. A transgenic gene therapy approach in mice resulted in a partial rescue of RTT phenotype when Mecp2 was expressed postnatally under an inducible promoter.

45. •- Guy J, Gan J, Selfridge J, et al.: Reversal of neurological defects in a mouse model of Rett syndrome. Science 2007, 315:1143-1147. This study showed a strong rescue effect in a Mecp2-deficient mouse with removable Stop codon. An improvement in neurological symptoms was observed with restored Mecp 2 expression, even for previously symptomatic mice, providing hope that RTT and other MECP2-associated disorders may be treatable after birth in humans.

46. Collins AL, Levenson JM, Vilaythong AP, et al.: Mild overexpression of $\mathrm{MeCP} 2$ causes a progressive neurological disorder in mice. Hum Mol Genet 2004, 13:2679-2689.

47. Moretti P, Levenson JM, Battaglia F, et al.: Learning and memory and synaptic plasticity are impaired in a mouse model of Rett syndrome. J Neurosci 2006, 26:319-327.

48. - Dani VS, Nelson SB: Intact long-term potentiation but reduced connectivity between neocortical layer 5 pyramidal neurons in a mouse model of Rett syndrome. J Neurosci 2009, 29:11263-11270. Recordings of layer 5 cortical pyramidal neurons in slices of Mecp2deficient mice showed that reduced excitatory synaptic connectivity, rather than the induction of long-term potentiation, was responsible for the decreased excitatory neurotransmission in RTT.

49. Kudo S, Nomura Y, Segawa M, et al.: Heterogeneity in residual function of $\mathrm{MeCP} 2$ carrying missense mutations in the methyl CpG binding domain. J Med Genet 2003, 40:487-493.

50. Kudo S, Nomura Y, Segawa M, et al.: Functional characterisation of MeCP2 mutations found in male patients with X linked mental retardation. J Med Genet 2002, 39:132-136.

51. - Zeev BB, Bebbington A, Ho G, et al.: The common BDNF polymorphism may be a modifier of disease severity in Rett syndrome. Neurology 2009, 72:1242-1247. (Published erratum appears in Neurology 2009, 73:161.) This study reported that a common polymorphism of BDNF, a known MeCP2 target gene, was significantly associated with severity in R168X MECP2mutant RTT patients, providing evidence for an independent genetic modifier effect. 